\title{
Development and Evaluation of a Solar Ultraviolet-B Radiometer
}

\author{
Shu TAKESHITA, Toshibumi SAKATA and Masako SASAKI \\ Tokai University \\ 1117 Kitakaname, Hiratsuka-shi, Kanagawa, 259-12
}

Paper originally published in Japanese in J.IEIJ. Vol.77-No.10, 1993

\begin{abstract}
A new band-spectral solar ultraviolet-B (UV-B: $290-320 \mathrm{~nm}$ ) radiometer was developed to achieve a simple and accurate outdoor measurement. The spectral response of the radiometer has a maximum at $305 \mathrm{~nm}$ and has a $20-\mathrm{nm}$ bandwidth. In this radiometer, incident solar UV-B irradiance is converted to visible irradiance by a phosphor layer and is detected by a Si-photodiode. The spectral, cosine, azimuth and temperature responses of the radiometer have been evaluated. Contamination by UV-A radiation longer than $320 \mathrm{~nm}$ in wavelength is less than $6 \%$ of the measured UV-B radiation. No changes in instrumental sensitivity nor in the spectral response of the radiometer have been found during periodical checks over a two-year span. Results indicate that this radiometer is useful for continuous, high time-resolution measurement of solar UV-B radiation.
\end{abstract}

KEYWORDS: azimuth response, cosine reponse, phosphor layer, solar ultraviolet-B irradiance, spectral response, temperature response, UV-B, iance, UV-B radiometer

\section{Introduction}

Recent observations of artificial satellites equipped with TOMS (Total Ozone Mapping Spectrometers) have confirmed that the ozone concentration in the stratosphere is decreasing ${ }^{1)}$, which has evoked anxiety that more and more solar ultraviolet-B (290-320 nm) irradiance may be reaching the earth. Measuring solar ultraviolet irradiance had attracted little attention until the ozone layer destruction became a reality. Solar irradiance had been measured in the past, but efforts were concentrated chiefly on the visible and infrared ranges ${ }^{2 / 3 / 4)}$ to meet the needs of solar energy conversion using a photovoltaic power system etc. There was no accurate radiometer to measure solar UV-B irradiance, and it had never been measured in Japan until 1990. In the process of our photochemical and photobiological investigations, we began studying physical methods to measure the ultraviolet irradiance around the wavelength of $300 \mathrm{~nm}$ simply and accurately ${ }^{5}$. In the 1980 's, when silicon photodiodes (Si-PD) with stable sensitivities in the visible range became commercially available, we stopped studying the direct method to measure ultraviolet irradiance, and started studying a new method, in which ultraviolet irradiance is converted, using a phosphor, to visible irradiance, which can be measured with a Si-PD. After selecting a phosphor we completed a radiometer designed to measure solar UV-B irradiance, and started a continual outdoor measurement in October 1990. We have already reported on solar UV-B irradiance measured on the earth's surface using this radiomter ${ }^{6 / 778}$. In this paper, we describe the results of various tests on the performance of the radiometer, which were carried out simultaneously during the continual measurement.

\section{Development of Solar UV-B Radiometer}

\subsection{Design Concept}

Minimum requirements for the solar UV-B radiometer are shown below.

1) A simple structure to measure wherever and whenever we choose.

2) Sensitivity for the range outside UV-B, with wavelengths longer than $320 \mathrm{~nm}$ must be lower by more than four figures, because of the extremely small quantity of UV-B reaching the earth's surface, less than $1 \%$ of total solar energy ${ }^{9)}$, and

3) Capability of continual outdoor measurement for one year.

To meet these requirements, first, we examine the method to separate UV-B irradiance from solar irradiance. Physical methods to measure ultraviolet irradiance are roughly classified into two types: spectrometry using a monochromator and band spectrometry using band-pass optical filters. Spectrometry using a monochromator has the advantage of excellent wavelength resolution, but it has poor time resolution due to wavelength scanning, and it is also expensive. Further more, it can not be transported 
easily because of it's restricted layout. Band spectrometry has the advantage of excellent time resolution, can be easily handled, and is less expensive. Comparing the two methods, we selected band spectrometry stressing time resolution and easy operation. Band spectrometry, however, has an unavoidable problem; there is no optical filter to measure UV-B irradiance with a rectangular spectral response in the UV-B range. Thus, instrumental sensitivity inevitably changes with the change of the spectral distribution of solar UV-B irradiance. This will be discussed in detail in Section 2.4.

\subsection{Detection of UV-B Irradiance}

Thermal and quantum detections are two physical methods to detect solar UV-B irradiance. Thermal detectors detect a temperature rise caused by thermal energy as an electric signal; a thermocouple is its typical thermal detector. Its response does not depend on the wavelength, which is advantageous, but it has poor sensitivity in detecting ultraviolet irradiance and is slow in response; therefore it is inappropriate for a continual measurement of weak solar UV-B irradiance.

Quantum detectors detect incident quantum energy as an electric signal by directly generating free electrons and electron-positive hole pairs. A phototube or a Si-PD is used as a typical photoelectric detector. A solar blind phototube designed to detect ultraviolet irradiance is superior to a Si-PD, eliminating visible and infrared irradiance that affect detection of UV-B irradiance. A Si$\mathrm{PD}$, however, is superior because of its smaller size and light weight. Thus, we chose a Si-PD. A Si-PD has a stable spectral response in the visible range, but it is low and unstable in the ultraviolet range ${ }^{10)}$. Further, the output is saturated with an extremely intense ultraviolet irradiance. Considering the above, we used a phosphor to transform solar UV-B irradiance into visible irradiance, which can be detected with high sensitivity by a Si-PD. Also, we combined an interference filter with the phosphor, which has absorption bands in the UV-B range. This combination allows detection of solar UV-B irradiance with an ideal rectangular spectral response between $290-320$ $\mathrm{nm}$ range. The spectral response of the radiometer is basically decided by the transmittance of the interference filter and the excitation spectrum of the phosphor used.

To realize an ideal response, the combination of the relative transmittance, $f_{\mathrm{IF}}(\lambda)$, of the interference filter and the relative excitation spectrum, $f_{p}(\lambda)$, of the phosphor was examined (Fig. 1). The spectral transmittance of the interference filter and the excitation spectrum of the phosphor layer are approximated by triangular bands with maximum wavelengths at the top and with a certain width at the bottom so that the following equation is derived:

$$
\mathrm{f}_{\mathrm{IF}}(\lambda+\Delta \lambda)=\mathrm{f}_{\mathrm{p}}(\lambda)
$$

When the maximum wavelength of the interference filter is equal to the maximum wavelength of the excitation
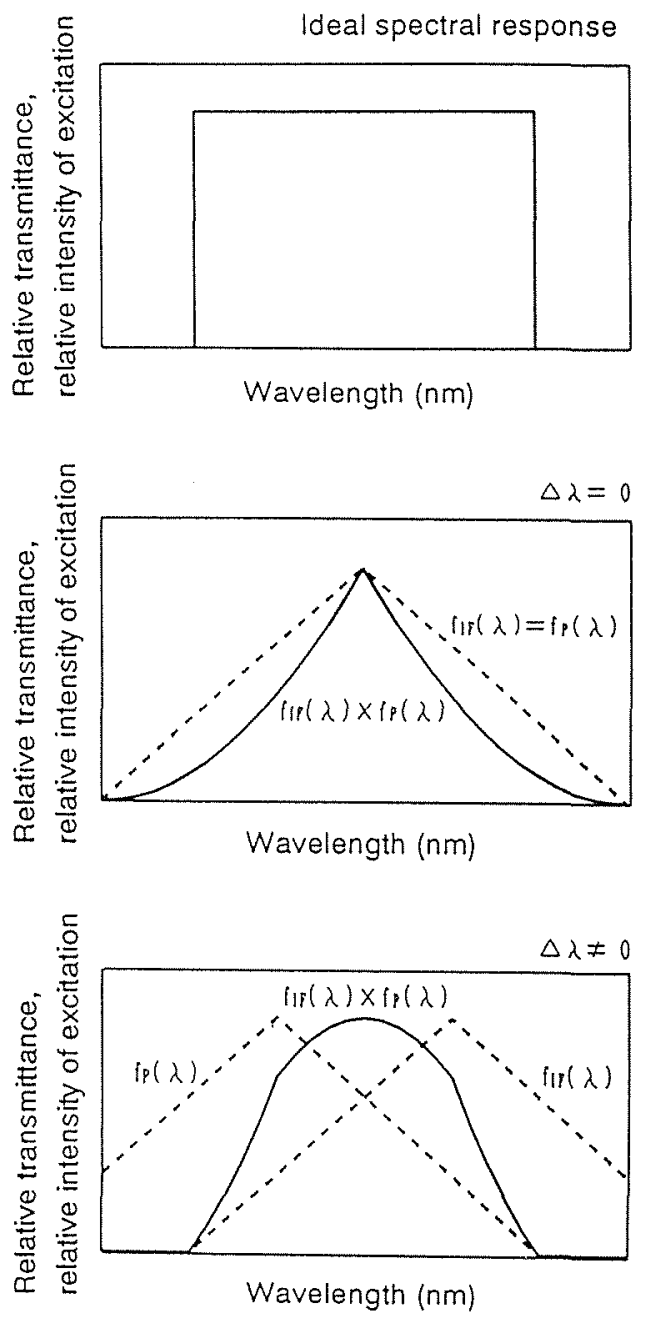

Fig. 1 Spectral response from the combination of the transmittance of the interference filter and the excitation spectrum of the phosphor

spectrum of the phosphor ( $\Delta \lambda=0$ : Fig. 1 , middle), the spectral response (solid line) forms a narrow band with a sharp peak. On the other hand, when the two maximum wavelengths are different ( $\Delta \lambda \neq 0$, Fig. 1 , bottom), the spectral response becomes rather rectangular, which is close to an ideal state (Fig. 1, top). With these results, we set the maximum wavelength of the interference filter and the maximum wavelength of the excitation spectrum of the phosphor at different values to make the spectral response close to a rectangle. The combination of the interference filter and the phosphor is a major factor in determining instrumental sensitivity, which is discussed in Section 2.4 .

Specifications for the interference filter were decided as follows: $313 \mathrm{~nm}$ for maximum wavelength transmitted, and $8.1 \mathrm{~nm}$ for the full width at half maximum (FWHM). Characteristics of the interference filter will be described in detail in Sections 2.3 and 2.4. We chose magnesium tungstate $\left(\mathrm{MgWO}_{4}\right.$, Toshiba) as the phosphor, which is a pure phosphor with high quantum efficiency ${ }^{11)}$. Fig. 2 shows excitation and fluorescence spectra of the phosphor. 


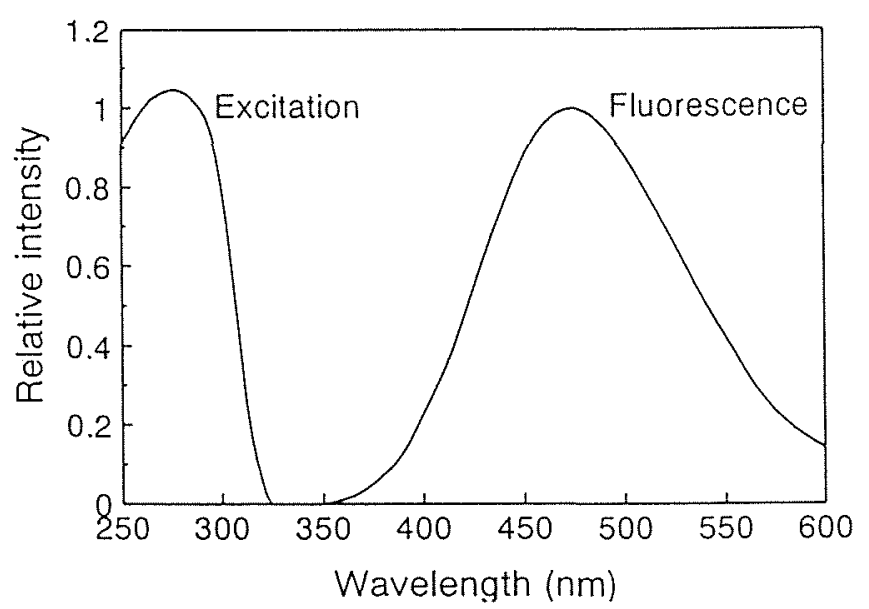

Fig. 2 Excitation and emission spectra of the phosphor

\subsection{Dependency of Interference Filter on Incident Angle}

Using an interference filter, the incident angle is an important factor. A large incident angle shifts the dominant wavelength to the shorter side ${ }^{121}$. Using this, we tried to increase the spectral response at shorter wavelengths. When increasing the incident angle, the spectral transmittance of the interference filter shifts to the short wavelength range. This is followed by a change in the spectral response. To make the incident angular distribution constant to the interference filter, we placed a diffuser in front of the interference filter. The increase of the spectral response in the short wavelength range and the change in the spectral response against the incidence angle were examined. An optical diffuser should have a surface of perfect diffuse transmittance. Thus, we selected an optical diffuser after actually installing it in the UV-B radiometer and measuring the spectral responses. We also checked the following factors: type of diffuser (quartz on one side \#280, quartz on both sides \#280 and Teflon), number of layers (1-3), distance between diffuser and photoelectric detector (Si-PD), and the incident angle against the radiometer. Results showed that the Teflon was the best. We obtained a stable spectral response after studying the thickness of the Teflon diffuser and the distance between the diffuser and the interference filter. Fig. 3 shows the spectral transmittance of the Teflon diffuser used in the UV-B radiometer.

\subsection{Simulation of the Instrumental Sensitivity}

It is impossible to make the spectral response of the UV$B$ radiometer perfectly rectangular. The change of instrumental sensitivity, $\mathrm{k}$, depending on the spectral distribution of solar irradiance which changes with time and

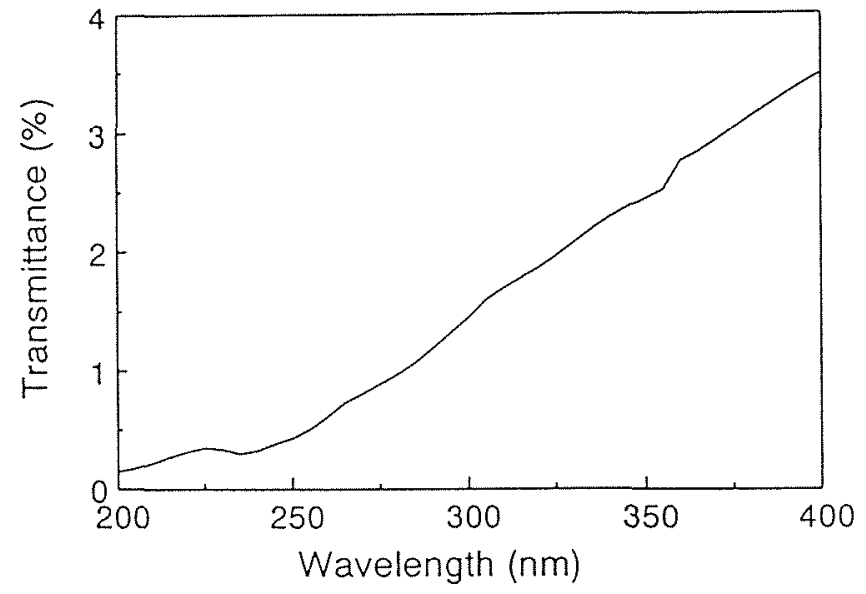

Fig. 3 Regular transmittance of the Teflon diffuser

atmospheric condition was simulated. The instrumental sensitivity is the constant when converting the output(electric current or voltage) of the UV-B radiometer into irradiance $\left(\mathrm{W} / \mathrm{m}^{2}\right)$ using the following equation:

$$
\begin{aligned}
& \text { Output of radiometer (A or V) } \int_{\text {vV-B }} I(\lambda) R(\lambda) d \lambda \\
& \mathrm{k}=\frac{}{\text { Irradiance of } U V-B\left(W / \mathrm{m}^{2}\right)}=\frac{}{\int_{U V-B} \mathrm{I}(\lambda) \mathrm{d} \lambda} \\
& \left(A W^{-1} m^{2}\right) \text { or }\left(\mathrm{VW}^{-1} \mathrm{~m}^{2}\right)
\end{aligned}
$$

Where, $I(\lambda)$ is the spectral irradiance of solar UV-B, $R(\lambda)$ is the spectral response of the UV-B radiometer, and $\lambda$ is the wavelength. In this equation, the requirement for constant instrumental sensitivity is spectral irradiance independent of wavelength, $\{\mathrm{I}(\lambda)=$ constant $\}$, or the spectral response of the UV-B radiometer independent of wavelength, $\{R(\lambda)=$ constant $\}$. Since these two conditions can not actually be met, the spectral response was estimated to achieve instrumental sensitivity as constant as possible.

Setting the maximum wavelength in a longer wavelength of UV-B, where the absorption by ozone has little effect, the variation of the instrumental sensitivity is minimal, however, increases in the spectral response of a longer wavelength outside UV-B, is not required. Changing the wavelengths, $310 \mathrm{~nm}, 305 \mathrm{~nm}$ and $300 \mathrm{~nm}$, at the maximum spectral responses, the change in instrumental sensitivity and the change in percentage of the output for outside UV$B$ against the total output of the radiometer were estimated. The spectral response was assumed to have a triangular waveform with FWHM of $14 \mathrm{~nm}$, and the solar spectral irradiance reaching the earth's surface was calculated using Iqbal's equation ${ }^{131}$. The following parameters were used for the calculation: total ozone $=300$ Dobson, precipitation 
$\AA$ of water $=2.0 \mathrm{~cm}, \AA$ Angstrom turbidity coefficient $\beta=0.1$, its exponent $\alpha=1.3$, ground albedo $=1.0$. Fig. 4 shows the results.

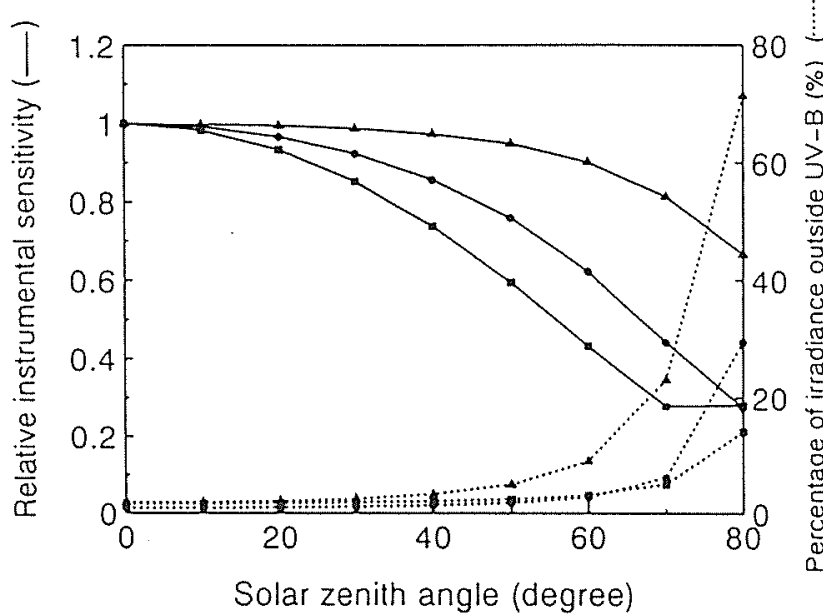

Fig. 4 Simulated dependency of relative instrumental sensitivity (-) and percentage of irradiance outside UV-B (.......) on solar zenith angle. wavelength with maximum responses of solar UV-B radiometer: $310 \mathrm{~nm}$ (4), $305 \mathrm{~nm}$ (수) and $300 \mathrm{~nm}$ (圈)
Instrumental sensitivities (solid line) are expressed by relative values, 1 for the solar zenith angle of 0 degree. When the peak wavelength of the spectral response is smaller $(300 \mathrm{~nm})$, the instrumental sensitivity rapidly decreases with the increase of the solar zenith angle.

On the other hand, the percentage of the output for irradiance outside UV-B against the total output, is almost independent of the peak wavelength of the response; the variation is within $10 \%$ below 60 degrees of the solar zenith angle, while it rapidly increases above 60 degrees.

With the peak wavelength of $305 \mathrm{~nm}$ of the spectral response, instrumental sensitivity is lower by $50 \%$ at a solar zenith angle of 70 degrees than at 0 degrees, and the output caused by irradiance outside UV-B increases to $10 \%$ against the total output. The equation above gives a UV-B irradiance of approximately $3.0 \mathrm{~W} / \mathrm{m}^{2}$ at a solar zenith angle of 0 degrees, and $0.13 \mathrm{~W} / \mathrm{m}^{2}$ at 70 degrees respectively; namely, UV-B irradiance at the solar zenith angle of 70 degrees is approximately $1 / 20$ less than the value at 0 degrees. Actually, the percentage of the data for a solar zenith angle of 70 degrees and above, is less than $34 \%$ at the solstitial point against the total, which is used to calculate daily integrated irradiance. This, when converted to irradiance, corresponds to approximately $2.5 \%$ of daily integrated irradiance. The variation of the instrumental
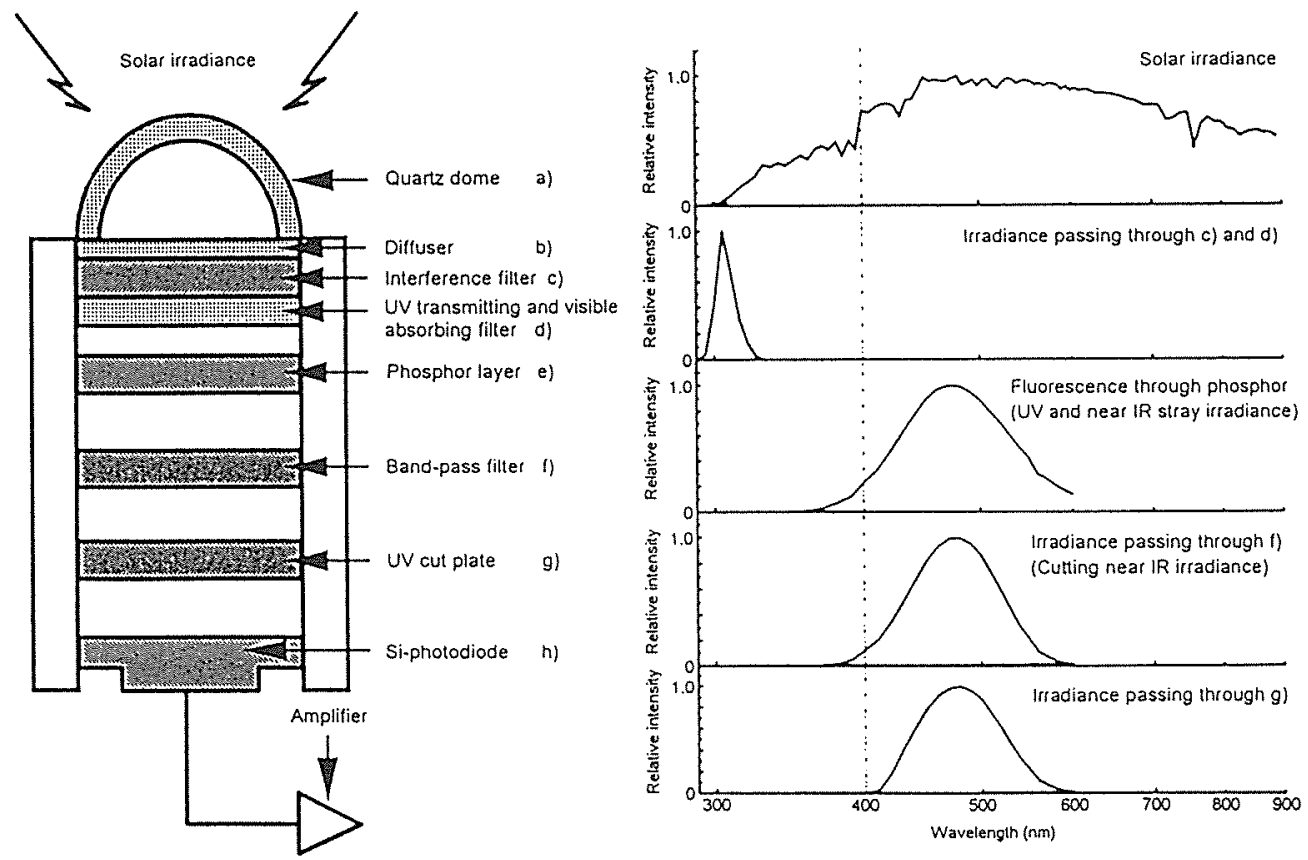

Fig.5 Optical components of the solar UV-B radiometer and spectrum conversion 
sensitivity, therefore, was estimated to cause no considerable error. With this background, a wavelength with a maximum spectral response was set at $305 \mathrm{~nm}$. The interference filter, described in Section 2.2, was based on data of the spectral response of the UV-B radiometer, the excitation spectrum of the phosphor, and the increased response in the shorter wavelength using the combination of the interference filter and the diffuser.

\subsection{Elimination of Stray Irradiance}

Two filters were combined to eliminate unwanted UV-A (320 - $400 \mathrm{~nm}$ : ultraviolet-A) and visible/near infrared stray irradiance. A fluorescence-free plastic plate (Fukubi) was used to eliminate UV-A stray irradiance. Most optical filters emit self-fluorescence, which makes a noise disturbing the fluorescence emitted from the phosphor, while Fukubi's plate cutting UV-A is free from it. A bandpass optical filter (B480, Hoya) was used to eliminate longer wavelengths of visible irradiance and near infrared stray irradiance.

\subsection{Optical Components of Solar UV-B Radiometer and Spectrum Conversion}

Results in Section $2.1-2.5$ were used to determine the optical components of the solar UV-B radiometer. Fig. 5 shows the components and the spectra converted from UVB irradiance through these components.

The components are briefly described below.

a) A transparent quartz dome to protect the diffuser from rain and dust

b) A Teflon diffuser (Nitias)

c) An interference filter to extract UV-B from solar irradiance

d) An optical band-pass filter (U340, Hoya) to eliminate weak visible irradiance through the interference filter and to pass ultraviolet irradiance

e) A phosphor layer $\left(\mathrm{MgWO}_{4}\right.$, Toshiba) to convert UV-B through interference filter into visible irradiance

f) An optical band-pass filter (B480, Hoya) to eliminate longer wavelength of visible and near infrared irradiance through the phosphor layer

g) A fluorescence-free plate (Fukubi) to cut UV-A and UV-B through the phosphor layer

h) A Si-PD (S1226-8BQ, Hamamatsu Photonics) to detect visible irradiance converted from UV-B irradiance

The completed detecting head is $10 \mathrm{~cm}$ in height, $18 \mathrm{~cm}$ in diameter, approximately $5 \mathrm{~kg}$, and $7 \mathrm{~cm}^{2}$ in lightreceiving area (Fig. 6). The output current from the Si-PD is amplified and converted to electric voltage.

Accordingly, continuous measurement of solar UV-B irradiance on the earth has become possible with this radiometer.

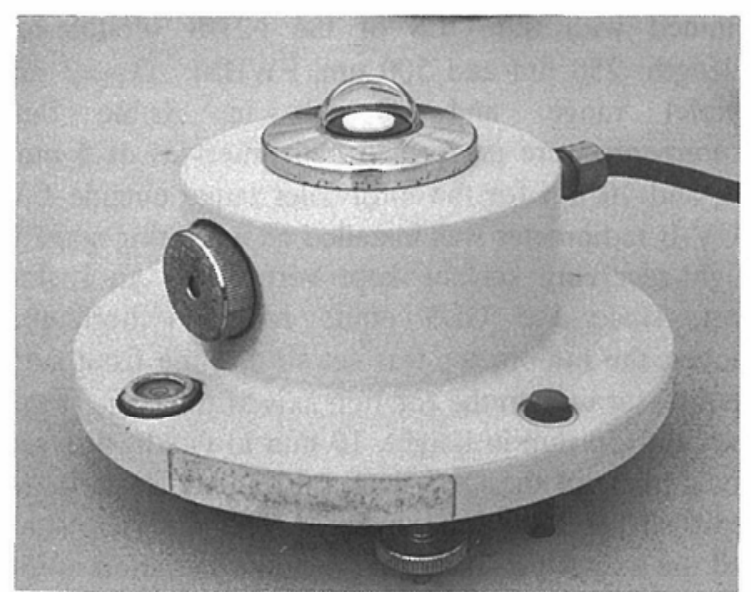

Fig.6 A photograph of the solar UV-B radiometer

\section{Performance and Evaluation of Solar UV-B Radiometer}

\subsection{Items for Measurement}

Six items below were measured to evaluate the solar UV$B$ radiometer.

a. Spectral response

b. Cosine response

c. Azimuth response

d. Temperature dependency

e. Blocking efficiency for the irradiance outside UV-B

f. Change with time

The light source used for items a, b, and f, was mostly monochromatic radiation from the Okazaki Large Spectrograph (OLS) of the National Institute for Basic Biology (NIBB) ${ }^{14)}$, a spectral radiator (CRM-FA, Nippon Bunko) for item c, continuous radiation from a $500 \mathrm{~W}$ Xenon short-arc lamp for item $\mathrm{d}$ and solar radiation for item e. Spectral response of the radiometer was determined with the OLS and a spectral irradiance standard lamp (JPD100-500-CS, Ushio, calibrated by Japan Electric Meters Inspection Co.) for item $\mathrm{f}$.

This UV-B radiometer has been used since October 1990 to measure solar UV-B irradiance continually. Since the performance test required approximately two weeks, the UV-B radiometer was taken away during this period interrupting the continual measurement. The test was repeated three times for item a (November 1990, November 1991, and November 1992), twice for item b (November 1990 and November 1991). For items $d$ and e, the test was done only once (February 1990). 


\subsection{Initial Performance and Evaluation}

\subsubsection{Spectral Response}

Spectral response in the range of $250-400 \mathrm{~nm}$ was determined with the OLS of the NIBB (double-blaze, wavelength: $250 \mathrm{~nm}$ and $500 \mathrm{~nm}$, FWHM: $\Delta \lambda_{1 / 2}=3 \mathrm{~nm}$ in ultraviolet range, and $10 \mathrm{~nm}$ in visible range). Measurements were performed with intervals of $5 \mathrm{~nm}$ for $\mathrm{UV}-\mathrm{B}$, and $10 \mathrm{~nm}$ for the ultraviolet range outside UV-B. The UV-B radiometer was installed on a rotating stage with the light-receiving surface kept vertical by an L-shaped support, since the OLS emits radiation horizontally. Moreover, the radiometer was set so that the front surface of the diffuser was on the rotation axis of the stage (Fig. 7). An exit slit ( $50 \mathrm{~mm}$ in height, $10 \mathrm{~mm}$ in width) was set on the focal plane of the OLS with a distance of $1 \mathrm{~m}$ between the exit slit and the radiometer. The diffuser was set behind the exit slit to make the incident radiation uniform, and an optical glass-filter was set behind the diffuser to cut secondary radiation from the grating.

Spectral irradiance was measured by a photon density meter (HK-1, Riken; hereafter called "photon meter") ${ }^{15)}$ for monochromatic lights equipped in the room. The photoelectric detector of the photon meter was a Si-PD (S1337-66BQ, Hamamatsu Photonics) calibrated by the Hamamatsu Photonics.

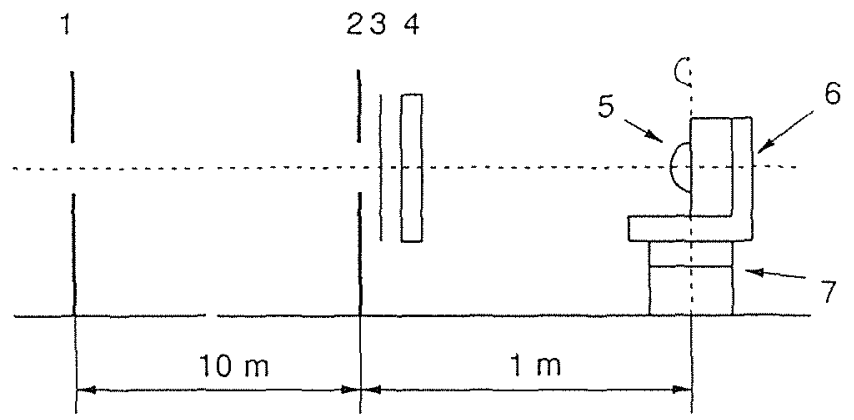

1. Projection window of the OLS

2. Exit

3. Diffuser

4. Filter

5. UV-B radiometer

6. L-shaped support

7. Rotaling stage

Fig. 7 Configuration to measure spectral and cosine responses

Radiation was measured with the photon meter replacing the solar UV-B radiometer. This replacement caused a time lag between the two measurements. To correct the light source variation caused by the time lag, the radiation at $345 \mathrm{~nm}$ was always monitored with a monitoring radiometer (Model 181, United Detector Technology). Outputs from the UV-B radiometer and the monitoring radiometer, or outputs from the photon meter and the monitoring radiometer, were measured at the same time with an analog digital conversion (A/D) board (ANALOG-PRO I, resolution: $0.2 \mathrm{mV}$, Canopus). At the third measurement (November 1992), the A/D board was replaced with a digital multimeter controlled by GP-IB (R6551, Advantest).
Relative spectral response of the solar UV-B radiometer is expressed by the following equation:

$$
\begin{aligned}
& \text { Relative } \\
& \text { spectral response }
\end{aligned}=\frac{\frac{\text { Output from UV-B radiometer }}{\text { Output from monitoring radiometer }}}{\frac{\text { Output from photon meter }}{\text { Output from monitoring radiometer }}}
$$

Outputs from the UV-B radiometer and the photon meter are divided by the output of the monitoring radiometer to obtain the relative outputs. Finally, the relative spectral response was calculated with the relative output from the solar UV-B radiometer divided by the relative output from the photon meter.

Wavelength at the maximum spectral response was measured to be $305 \mathrm{~nm}$. Fig. 8 shows measured relative spectral responses which were normalized at $305 \mathrm{~nm}$. The response at $400 \mathrm{~nm}$ was lower by more than five digits than the maximum response as shown in this figure.

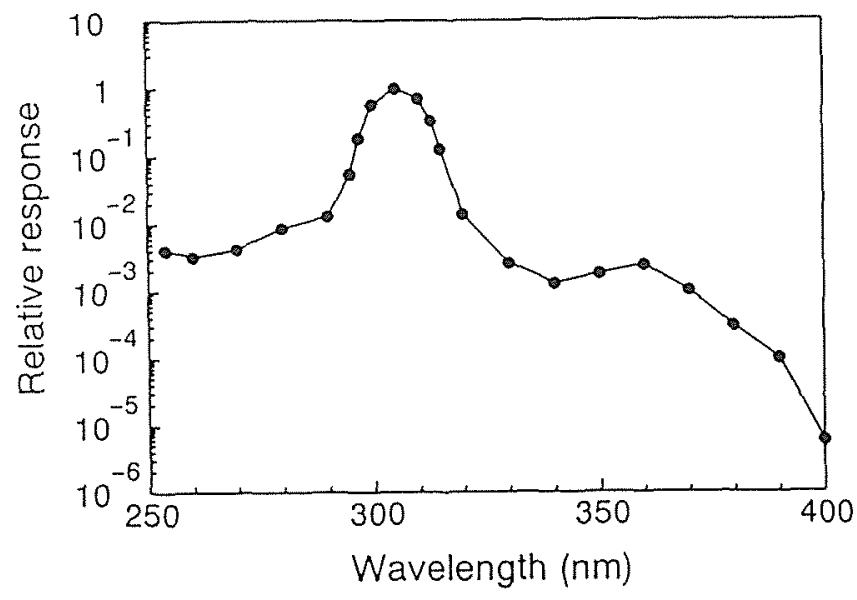

Fig.8 Measured spectral response

\subsubsection{Cosine Response}

Cosine response was measured with 10-degree intervals, using a fixed horizontal beam of light at $305 \mathrm{~nm}$ from the OLS. The UV-B radiometer was in a vertical position turned around a vertical axis. Relative outputs from the UV-B radiometer were obtained by dividing the actual output divided by output from the monitoring radiometer. The observation results are shown in Fig. 9.

The deviation from an ideal cosine curve was $0.99 \%$ at the incident angle of 10 degrees, $1.49 \%$ at 30 degrees, $-4.76 \%$ at 50 degrees, $-5.35 \%$ at 60 degrees, and $6.91 \%$ at 80 degrees respectively. When comparing these results with the JIS $\mathrm{Cl} 1609$ specification [Illuminance Meter] ${ }^{(6)}$, the radiometer was found to meet the general AA-grade. The radiometer was also found to meet the first class specification, when compared to the WMO (World Meteorological Organization) specification ${ }^{17)}$ for a pyranometer. Since the main portion of solar UV-B irradiance is the diffuse component, it may considerably decrease error. 


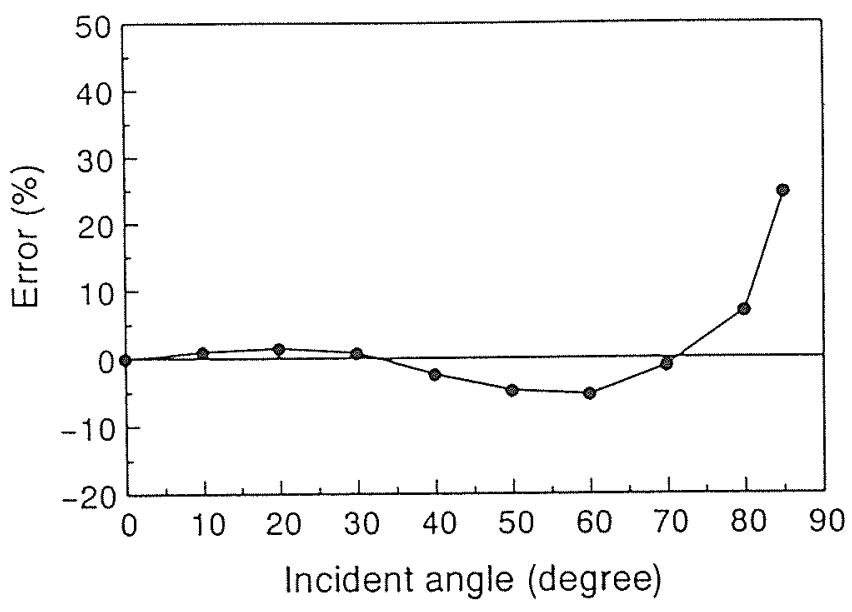

Fig. 9 Measured cosine response

\subsubsection{Azimuth Response}

Azimuth response was measured using $305 \mathrm{~nm}$ monochromatic radiation (FWHM: $\Delta \lambda_{1 / 2}=14 \mathrm{~nm}$ ) from a spectral radiator (CRM-FA, Nippon Bunko) as a light source, using an A/D conversion board (ANALOG-PRO I, resolution: $0.24 \mathrm{mV}$, Canopus). The incident angle was set to 80 degrees (Altitude of the sun: 10 degrees) and the incident azimuth angle was changed from 0 to 360 degrees with intervals of 60 degrees. The results are shown in Fig. 10.

Errors were within $+/-5 \%$ in the range of $0-360$ degrees. This corresponds to the first class specification of the WMO for a pyranometer.

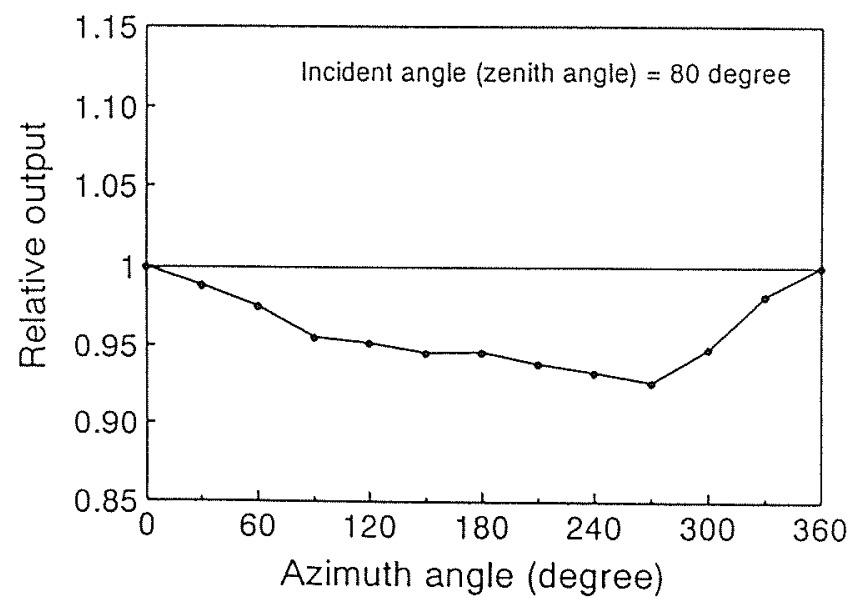

Fig. 10 Measured azimuth response

\subsubsection{Temperature Dependency}

Temperature dependency was measured as follows:

For a temperature range lower than room temperature, the solar UV-B radiometer was chilled in a freezer to $-30{ }^{\circ} \mathrm{C}$.
The radiometer was the taken out of the freezer, and outputs and temperatures were measured at the same time as the temperature increased. For a temperature range higher than room temperature, the radiometer was heated in an oven at $70{ }^{\circ} \mathrm{C}$. The radiometer was taken out, and outputs and temperatures were measured at the same time, as the temperature decreased. The light source was a 500 W Xenon short-arc lamp. We eliminated irradiance under $290 \mathrm{~nm}$, using an UV-transmitting filter (UV29, Toshiba), contained in a Xenon short-arc lamp emission but not in solar irradiance reaching the earth's surface. Temperature was measured on the side of the radiometer using a digital contact thermometer (Type 2573, Yokogawa). The variation of the light source was monitored using an ultraviolet radiometer commercially available (UVR-36, Topcon).

Temperature dependency of the solar UV-B radiometer was determined to be $0.2 \% /{ }^{\circ} \mathrm{C}$ in the range of $-10-+50{ }^{\circ} \mathrm{C}$. In an actual measurement, assuming that the maximum temperature difference between test sites is $40{ }^{\circ} \mathrm{C}$, an error of $8 \%$ will result. We added a compensation circuit to the radiometer, so that the output variance became lower than $1 \%$ between 0 and $40^{\circ} \mathrm{C}$.

\subsubsection{Blocking Efficiency for Irradiance Outside UV-B}

An opaque tube, $4.0 \mathrm{~cm}$ in diameter and $28 \mathrm{~cm}$ in height, was vertically equipped on the light-receiving surface of the UV-B radiometer, so that only scattered solar irradiance from the limited zenith angle can penetrate into the UV-B radiometer. On top of this tube, various sharp cut filters (UV32, UV34, UV36, L38, LA0 and LA2, Hoya; UV35, L37 and L39, Toshiba) were placed to change the wavelength range of incident solar irradiance, and the contamination was measured and analyzed. The zenith angle of the sun was approximately 44 degrees (air mass $=1.38$ ) and the weather was fine.

Table 1 shows percentages of contamination against the total output. The contamination was measured to be less than $6 \%$ and could detect irradiance longer than $370 \mathrm{~nm}$.

Table 1 Percentage of contamination outside the UV-B range

\begin{tabular}{lccl}
\hline Filter & $\begin{array}{c}\text { Cut-off } \\
\text { wavelength } \\
(\mathrm{nm})\end{array}$ & $\begin{array}{c}\text { Output ratio } \\
(\%)\end{array}$ & Time \\
\hline L42 & 420 & 0 & $13: 08$ \\
L40 & 400 & 0 & $13: 09$ \\
L39 & 390 & 0 & $13: 09: 30$ \\
L38 & 380 & 0 & $13: 10$ \\
L37 & 370 & 0 & $13: 11$ \\
UV36 & 360 & 1 & $13: 11: 30$ \\
UV35 & 350 & 1 & $13: 12$ \\
UV34 & 340 & 1 & $13: 12: 30$ \\
UV33 & 330 & 5 & $13: 13$ \\
UV32 & 320 & 6 & $13: 14$ \\
\hline
\end{tabular}




\subsubsection{Continual Measurement and Evaluation}

Relative spectral responses of the UV-B radiometer were measured, using the OLS as a light source, at two months (November 1990), one year, and two years after starting the outdoor measurement to calculate and compare instrumental sensitivities (Fig. 11). The wavelengths for the maximum relative spectral response, obtained in the three measurements, coincided perfectly. As shown in Fig. 11, the instrumental sensitivity varied by less than $+1-$ $6 \%$ in the two years, with the solar zenith angles of $0-80$ degrees. The instrumental sensitivity was calculated using the spectral solar irradiances described in Section 2.4.

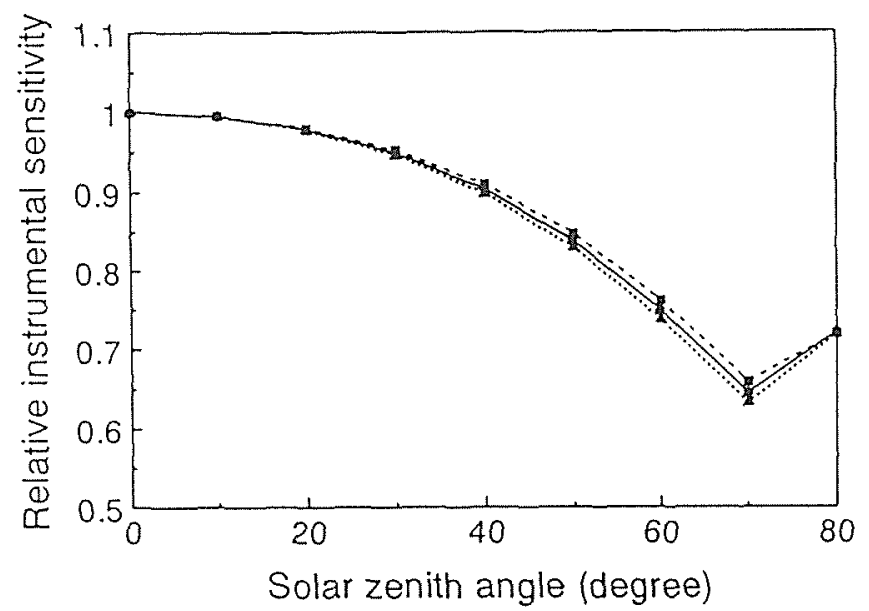

Fig. 11 Instrumental sensitivities reduced from the measured spectral responses for 1990

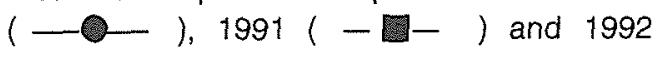
$(\ldots \Delta \ldots)$

The instrumental sensitivity was also measured independently in November 1991 and in November 1992 using a spectral irradiance standard lamp (JPD-100-500CS) as a light source. The comparison of the data gave a difference of $-2 \%$ for 1992 compared to 1991.

With these results, we concluded that the spectral response and instrumental sensitivity of the solar UV-B radiometer did not change during the continual two year test with certain conditions taken into consideration: layout in measuring relative spectral response in the former, and reproducibility of spectral irradiance in the later.

\section{Discussion}

The measurements and evaluation described in Section 3 show that the new solar UV-B radiometer meets all requirements. However, two problems exist: the variation of instrumental sensitivity depending on solar spectral irradiance and insufficient blocking of radiation longer than $320 \mathrm{~nm}$. These problems are discussed below.

Because of non-rectangular spectral response in the UV-B range, instrumental sensitivity changes with the change in the solar spectral irradiance. We further studied the achieved level of instrumental sensitivity simulated in Section 2.4. As seen in Fig. 11, instrumental sensitivity decreased with the increase in the solar zenith angle, and at 70 degrees (air mass $=3$ ), the sensitivity became lower by $30 \%$. This decrease is smaller than the value simulated in Section 2.4, and the spectral response of the instrument was shown to be close to the goal. With a constant instrumental sensitivity, however, the UV-B measured irradiance is lower than the true value by $30 \%$ at a solar zenith angle of 70 degrees, which should be corrected to an instantaneous value. When measured UV-B irradiance is used as a part of the daily total, there may be no problem as mentioned in Section 2.4.

In Section 3.2.5, radiation outside the UV-B range was measured to be $6 \%$, which is less than the level (below $10 \%)$ estimated in Section 2.4. When the solar zenith angle is higher than 70 degrees (air mass $\geqq 3$ ), and the percentage of contamination is estimated to be much higher than $10 \%$, we may need further correction.

In summary, solar UV-B irradiance measured, with an instantaneous value or a daily total, needs no correction for a solar zenith angle lower than 70 degrees (air mass $\leqq 3$ ). Even at a solar zenith angle above 70 degrees (air mass > 3 ), no correction is needed when the data is treated as a part of the daily total. This is because the UV-B irradiance itself is small and the expected error is negligible. The instrumental sensitivity and the irradiance outside the UV-B range should be corrected, however, when they are used as instantaneous values. We are now studying the correction method.

\section{Conclusion}

Since solar UV-B irradiance measurement has not been considered as important as UV-A and UV-C measurements, only a few radiometers for the UV-B range, including the sunburning ultraviolet meter ${ }^{18}$, have been commercially available. This paper has described the performance and the evaluation results regarding the new solar UV-B radiometer developed under these circumstances.

1. The wavelength for the maximum spectral response is $305 \mathrm{~nm}$, and the response at $400 \mathrm{~nm}$, which is outside the range of UV-B, is lower by more than five orders of magnitude.

2. The cosine response is in accordance with the JIS specification for a general AA class illuminance meter, and with the WMO specification for a first class pyranometer.

3. The azimuth response is in accordance with the WMO specification for a first class pyranometer.

4. The temperature dependency is within $1 \%$ for temperatures between 0 and $40^{\circ} \mathrm{C}$.

5. The contribution of radiation outside the UV-B range is less than $6 \%$.

6. During the two years of continual outdoor measurement, the spectral response was constant. 
7. The values obtained through the measurement with this radiometer need not be corrected based on instrumental sensitivity and percentage of contamination, as long as they are handled as daily totals. On the other hand, correction is needed when they are handled as instantaneous values and with the solar zenith angle of more than 70 degrees; the correction method is now being studied.

Thus, this radiometer is considered as being applicable to real-time continual measurement of solar UV-B irradiance. Hoping this radiometer will serve to measure and continually analyze UV-B irradiance as well as determine the biological fatal dose, we will continue to make efforts to improve it further. A calibrating method in the UV-B range must be developed, since there is no standard method in Japan and/or abroad.

This study was supported by a number of people: $\mathrm{Mr}$. Okada of Koshin Industries Ltd. for the interference filter, Mr. Sugimoto of Toshiba Lighting \& Technology Co. for the phosphor, Messrs. Miyake and Nakamura of EKO Instrument Trading Co. for preparing the UV-B radiometer, and Messrs. Watanabe and Kubota of NIBB, for the Performance test of the radiometer. This research was supported in part by the Science Research Promotion Fund from the Japan Private School Promotion Foundation (1989-1991), and also by the National Institute for Basic Biology Cooperative Research Program of the Okazaki Large Spectrograph (Subject Numbers: 88-517, 89-512, 90524, 91-505, and 92-503 for 1988-1992). We would like to express our gratitude to them.

\section{References}

1) Stolarski, R. S. et al.: Total Ozone Trends Deduced from Nimbus 7 TOMS Data. Geophy. Res. Lett. 186 (1991) 1015-1018.

2) Habu et al.: Measurement of the Solar Spectral Irradiance at Tanashi, Tokyo (I):

Researches of the Electrotechnical Laboratory, No. 812 (1981).

3) Habu et al.: Measurement of the Solar Spectral Irradiance at Tanashi, Tokyo (II):

Researches of the Electrotechnical Laboratory, No. 813 (1981).

4) Habu et al.: Measurement of the Solar Spectral Irradiance at Tanashi, Tokyo (III):

Researches of the Electrotechnical Laboratory, No. 830 (1983).
5) Ohsawa et al.: Optical Radiometry: Reliability of Detectors for Practical Use. Proc. Soc. Photograph Sci. and Tech. Jpn., (1983), p.130.

6) Takeshita et al.: Solar UV-B measurements by TokaiSolar Radiation Monitoring System. Proc. Illum. Engng. Inst. Jpn., (1991), p.204.

7) Takeshita et al.: Solar UV-B Measurements by TokaiSolar Radiation Monitoring System II. Proc. Illum. Engng. Inst. Jpn., (1992), p.152.

8) Sasaki, M. et al.: Ground-Based Observation of Biologically Active Solar Ultraviolet-B Irradiance at $35^{\circ} \mathrm{N}$ Latitude in Japan. J. Geomag. Geoelectr. 45 (1993) 473-485.

9) CIE: Recommendations for the Integrated Irradiance and the Spectral Distribution of Simulated Solar Radiation for Testing Purposes. CIE Publ. No. 20 (TC-2.2), (1972).

10) Nakagawa, Y, et al.: Change with Parage of Time on Silicon Photodiodes Spectral Responsivity. Proc. Illum. Engng. Inst. Jpn., (1990) p. 127.

11) Narita, K.: Relative Quantum Efficiency of Various Lamp Phosphors. J. Illum. Engng. Inst. Jpn. 69-2 (1985) 65-69.

12) Heavens, O. S. and Dennison, D. M.: Reflection and Transmission Interference Filters Part I. Theory. J. Opt. Soc. Am. 37 (1947) 451-465.

13) Iqbal, M.: An Introduction to Solar Radiation, Academic Press (1983) p.107-168.

14) Watanabe, M. et al.: Design and Performance of the Okazaki Large Spectrograph for Photobiological Research. Photochem. Photobiol. 36 (1982) 491-498.

15) Hashimoto, $T$. et al.: A High-Sensitivity Photon Density Meter for Monochromatic Lights. Abstracts of the Ann. Meeting, Jpn. Soc. Plant Physiol., (1982), p.38.

16). JIS C1609-1993 (Illuminance Meter).

17) WMO: Guide to Meteorological Instruments and Methods of Observation. World Meteorological Organization, (1985)

18) Berger, D. S.: The Sunburning Ultraviolet Meter: Design and Performance. Photochem. Photobiol. 24 (1976) 587-593. 